\title{
Clinical Study \\ Reactive Lymphoid Hyperplasia of the Liver: A Clinicopathological Study of 7 Cases
}

\author{
Lei Yuan, Youlei Zhang, Yi Wang, Wenming Cong, and Mengchao Wu \\ Second Department of Hepatic Surgery, Eastern Hepatobiliary Surgery Hospital, Second Military Medical University, \\ 225 Changhai Road, Shanghai 200438, China
}

Correspondence should be addressed to Yi Wang, wangyi-ehbh@163.com

Received 9 February 2012; Accepted 22 June 2012

Academic Editor: Giuliano Testa

Copyright ( 2012 Lei Yuan et al. This is an open access article distributed under the Creative Commons Attribution License, which permits unrestricted use, distribution, and reproduction in any medium, provided the original work is properly cited.

Background. Reactive lymphoid hyperplasia (RLH) of the liver is a benign focal liver mass that may mimic a malignant liver tumor. Although rarely encountered in clinical practice, it often poses diagnostic and management dilemmas. Methods. Cases diagnosed as hepatic RLH between January 1996 and June 2011 were investigated in a retrospective study. Clinicopathological features as well as follow-up information of the cases were studied. Results. A total of seven cases of hepatic RLH were investigated, with a median age of 46 years (range: 33-76 years). Hepatic RLH was accompanied by concomitant diseases in some patients. The average size of hepatic lesions of our cases was $45 \mathrm{~mm}$ (range: 15-105 mm). All of the cases were not accurately diagnosed until confirmed by pathological findings, and surgical resections were performed for all. Postoperative course was uneventful for all of the patients during followup. Conclusions. RLH of the liver is a rare benign disease with a female predilection of unknown etiology. It is very difficult to correctly diagnose this disease without pathological results. Subtle differences on radiological findings of it may be helpful for differential diagnosis from other diseases. Curative resection of the lesion is suggested for the treatment of this disease.

\section{Introduction}

Reactive lymphoid hyperplasia (RLH), also termed as nodular lymphoid lesion $[1,2]$ or pseudolymphoma [3-8], is a rare benign condition which forms a liver mass typically infiltrated by massive heterogeneous mature lymphoid cells without prominent nuclear atypia, with formation of follicles and germinal centers. Up to now, there have been only scattered case reports about hepatic RLH [1-24]; the paucity of information about its clinicopathological features poses diagnostic and management dilemmas. Manifested as a focal liver mass, hepatic RLH may mimic a malignant liver tumor, often giving rise to misdiagnosis. Therefore, we reviewed our experience in a series of 7 patients with hepatic RLH who were treated by surgical resection. This was the largest series of cases of this disease so far, and a detailed evaluation of its clinical features, radiologic characteristics, and pathologic findings will assist in future diagnosis of this disease.

\section{Methods}

The data of all cases diagnosed as hepatic RLH or pseudolymphoma and treated by surgery in Eastern Hepatobiliary Surgery Hospital, a tertiary university hospital in China, between January 1996 and June 2011 were obtained from the computerized files. Results of imaging studies such as ultrasonography (US), computerized tomography (CT), and magnetic resonance imaging (MRI), the clinicopathological findings and the outcomes of followup of the patients were retrospectively reviewed.

All the patients underwent surgery with complete resection of lesion(s). The resected specimens were fixed in $4 \%$ buffered formalin, processed, and embedded in paraffin. Histologic sections were stained with hematoxylin and eosin. Immunohistochemistry of paraffin sections was carried out using EnVision system (a two-step staining technique) as described previously [25]. Negative control slides omitting 


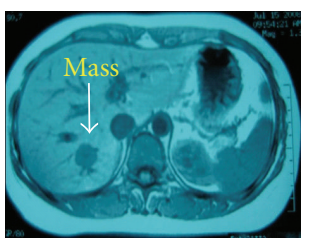

(a)

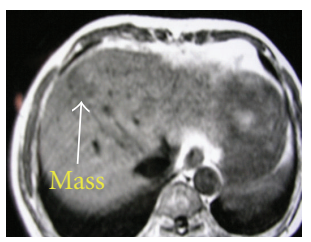

(f)

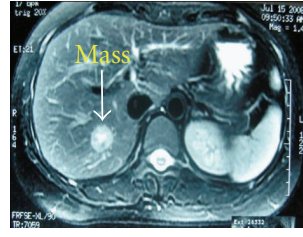

(b)

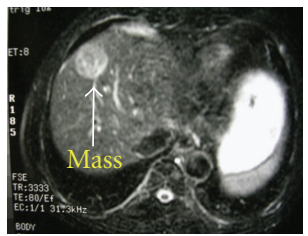

(g)

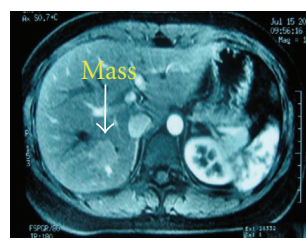

(c)

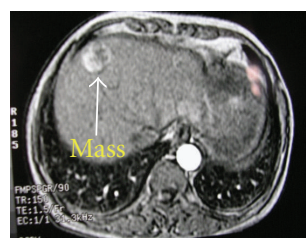

(h)

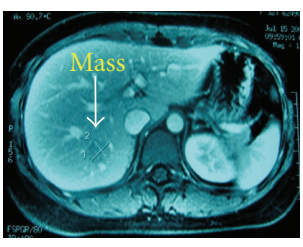

(d)

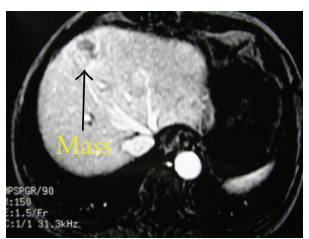

(i)

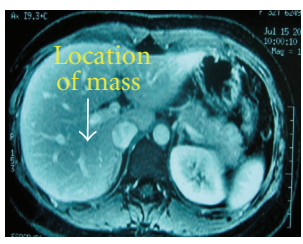

(e)

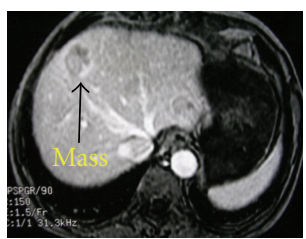

(j)

FIGURE 1: Comparison of appearances of hepatic RLH (a-e) with hepatocellular carcinoma ( $\mathrm{f}-\mathrm{j}$ ) on fast spoiled gradient recalled echo (FSPGR) MRI. (a) On unenhanced T1-weighted image, the lesion is hypointense signal relative to normal liver parenchyma. (b) The lesion is hyperintense signal in the same location on T2-weighted image. (c) The lesion is enhanced in the arterial phase. (d) The lesion is hypodense in the portal phase. (e) The lesion is unclear in the delayed phase. (f) On plain T1-weighted imaging scan, the lesion is hypointense signal relative to normal liver parenchyma. (g) The lesion is hyperintense signal in the same location on T2-weighted image. (h) The lesion is significantly enhanced in the arterial phase. (i) The lesion is hypodense in the portal phase. (j) The lesion became more hypodense in the delayed phase.

the primary antibodies were included in all assays. The evaluation of the immunohistochemical findings was performed without any knowledge of the clinicopathologic data by two independent clinical pathologists.

After surgery, all the patients were monitored by serumtumor markers, abdomen US, and chest X-ray every 1 to 3 months. If recurrence suspected, the patients underwent CT or MRI for further evaluation. The last followup was completed on December 31st, 2011 and the data were analyzed after the last followup.

\section{Results}

3.1. Patients' Demographics. From January 1996 to June 2011, a total of 45,000 patients with occupied lesion(s) in liver were admitted to our hospital for partial hepatectomy. In 7 of them, hepatic RLH was confirmed by pathological examination of resected specimens. There were 6 females and 1 male, with a median age of 46 years (range: 3376 years). The patients were either asymptomatic or had mild nonspecific symptoms such as abdominal discomfort. Hepatitis B surface antigen was negative in 6 female patients, but positive in the male patient who was accompanied by cirrhosis. Hepatitis $\mathrm{C}$ virus antibody was negative in all the patients. Tumor markers including AFP, CEA, and CA199 were within normal range in all cases. Hepatic function tests and blood routine test were normal. Hemangiomas happened to occur simultaneously with RLH in two patients, and gall bladder calculi and fibroadenoma of breast are found in two female patients. In this series, the sizes of hepatic lesions varied from $13 \times 15 \mathrm{~mm}$ to $105 \times 65 \mathrm{~mm}$ on US scan, and the lesion of the male patient progressed during three years before operation (from $1.0 \times 1.2 \mathrm{~cm}$ to 4.0 $\times 2.0 \mathrm{~cm}$ ). Preoperatively, hepatocellular carcinoma (HCC) was suspected in 6 patients and intrahepatic cholangiocellular carcinoma in the other one.

3.2. Surgical Outcomes. All the patients underwent curative local resections, and the postoperative course was uneventful in all cases. After surgery, no patient received subsequent therapy for hepatic RLH. Recurrence did not occur in any of our patients during a median followup of 68 months (range: 6-228 months).

3.3. Features on Imaging Studies. All the lesions of RLH were revealed as well-defined hypoechoic masses on US examination. The lesions were presented as hypodense masses on plain CT scan, slightly enhanced in the arterial phase, hypodense in the portal phase and the delayed phase as compared with liver parenchyma. MRI depicted hepatic RLH as nodular lesions with hypointense signal masses on T1-weighted imaging (Figure 1(a)), hyperintense signal on T2-weighted imaging (Figure 1(b)), and the lesions were moderately enhanced in the arterial phase (Figure 1(c)) followed by hypodense areas in the portal phase (Figure $1(\mathrm{~d})$ ) and unclear in the delayed phase (Figure 1(e)).

3.4. Pathologic and Immunohistochemical Analysis. On the cut sections, the lesions were grossly distinct firm nodules well demarcated from the surrounding liver tissue, sometimes encapsulated (5/7), with small areas of hemorrhage and necrosis (3/7) (Figure 2(a)). Histologic findings were similar for all the patients. Seen as a well-delineated nodular area microscopically, the lesions comprised a massive infiltration of heterogeneous mature lymphoid cells with no prominent nuclear atypia (Figure 2(c)), forming follicles (varied in size and shape) and germinal centers (Figure 2(b)), and there was no evidence of a monoclonal $\mathrm{B}$ cell population. 


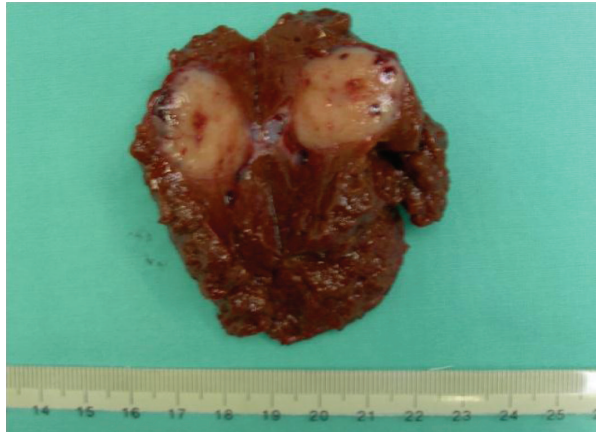

(a)

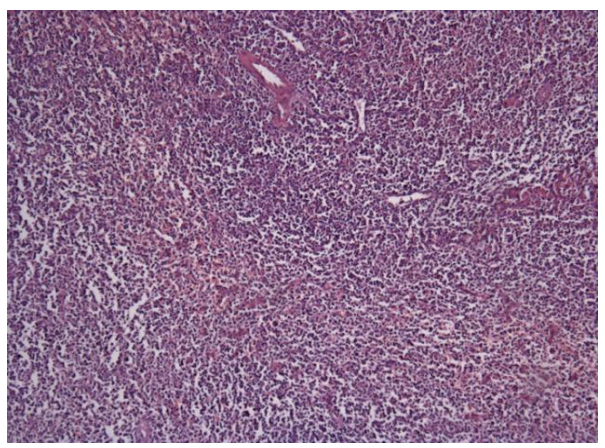

(c)

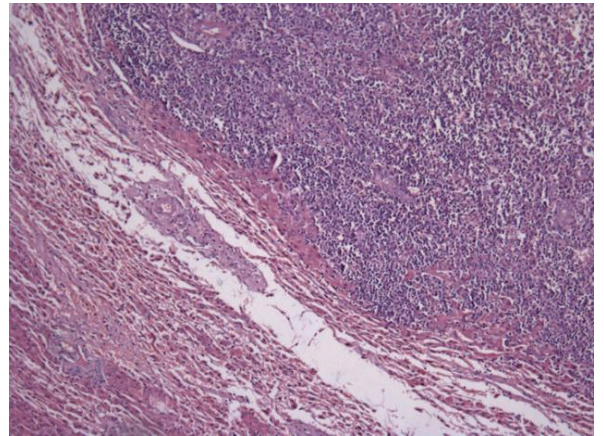

(b)

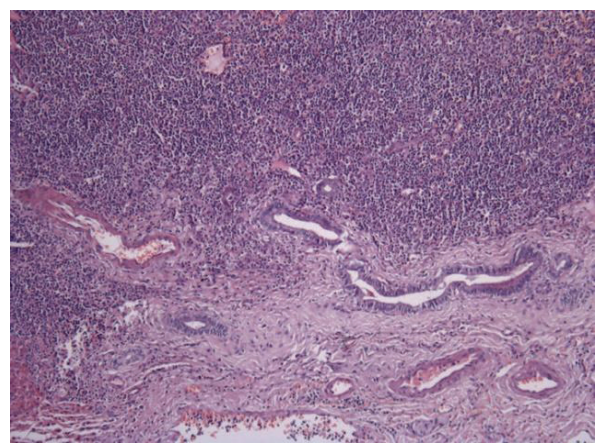

(d)

FIgURE 2: (a) Macroscopically, a cut section of the resected liver showed a well-circumscribed, encapsulated, yellow-white nodular lesion in segment 6, with small areas of hemorrhage and necrosis. (b) Microscopically, the lesion was well demarcated and encapsulated, and comprised a massive infiltration of mature lymphoid cells, forming follicles and germinal centers (H\&E staining, 100x magnification). (c) The infiltrated lymphoid cells was mature and heterogeneous, with no nuclear atypia or polymorphism (H\&E staining, 100x magnification). (d) Note the lymphocytic infiltration in the portal tracts around the lesion (H\&E staining, 100x magnification).

In addition, fibrous materials aggregating in parts of the lesions (4/7), lymphocytic infiltration in the portal tracts around the nodular lesion (5/7) (Figure 3(d)), and bile ductules $(2 / 7)$ were seen within the lesion. Germinal centers mainly comprised CD20 (+) and LCA (+) lymphocytes (Figures 3(a) and 3(b)), while lymphocytes in the interfollicular area and surrounding germinal centers were CD3 $(+)$ and/or CD45RO (+) (Figures 3(c) and 3(d)). A polyclonal pattern of IgH (immunoglobulin heavy chain) and TCR- $\gamma$ (T-cell receptor gamma) gene rearrangements of lymphocytes in the lesion was found in most patients.

\section{Discussion}

RLH is a benign condition that may occur, besides in liver, in the gastrointestinal tract [26], orbit [27], lung [28], skin [29], and thyroid [30]. Hepatic RLH was first reported by Snover et al. in 1981 [8], and only 34 cases have been reported in the English literature until now [1-25, 31,32]. We found 7 cases from 45,000 patients who underwent hepatectomy for liver masses, indicating the rarity of this disease. The incidence rate of hepatic RLH seems to be discovered increasing with time, as shown in Figure 4, perhaps for the advances in diagnostic imaging technologies and the accumulation of doctors' experience. However, the incidence rate might be underestimated due to doctors' limited understanding of this disease.

At present, this is the largest case series review of hepatic RLH. In total, 41 cases of hepatic RLH are reported, including 36 females and 5 males (median age of 57 years; range, 15-85 years). All hepatic RLH patients were adults except for one 15-year-old female. A female predilection of this disease was obvious in both the present series (6/7) and when considering the sum of all cases (36/41). 6 cases have multiple hepatic lesion of RLH, and there were 49 hepatic lesions altogether. The average size (in greatest dimension) of hepatic lesions of our cases was $45.0 \mathrm{~mm}$ (range: 15$105 \mathrm{~mm}$ ), which was bigger than that $(15.6 \mathrm{~mm}$ in average; range: $4-60 \mathrm{~mm}$ ) of the reported cases. The background data and the clinical characteristics of these 41 patients were summarized in Table 1.

The exact etiology of this disease remains unclear. An association between the development of hepatic RLH and some disease such as autoimmune diseases $[1,4-7,9,11]$ and malignant tumors $[3,6,10,13,15,20,22,31]$ has been suggested to be most likely involved in previous reviews. However, we believe this is not convincing because such concomitant diseases were not found in this series, and the reviews are independent, intermittent case reports.

It is very difficult to make a correct diagnosis of hepatic RLH before operation, as almost all of the cases including 


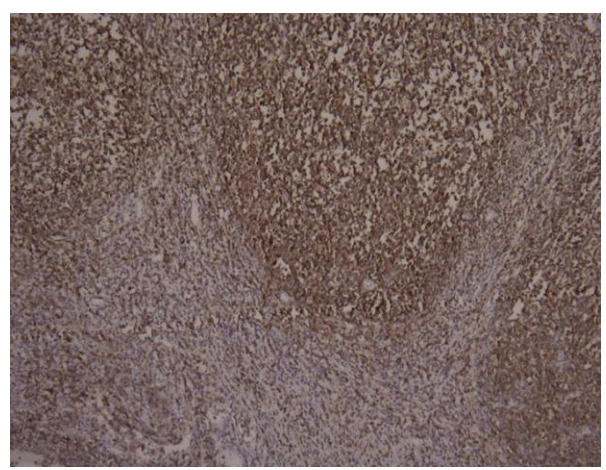

(a)

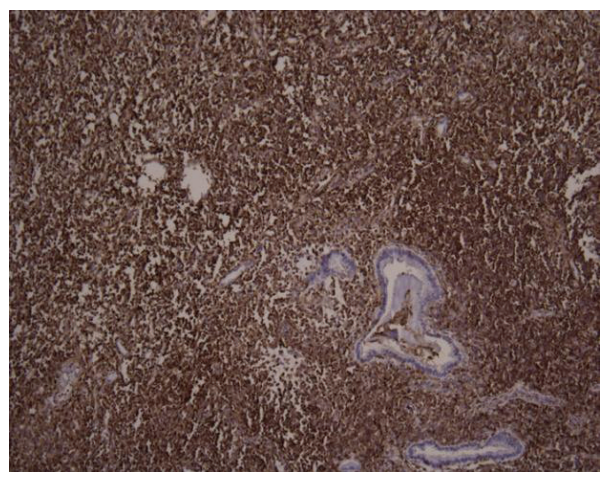

(c)

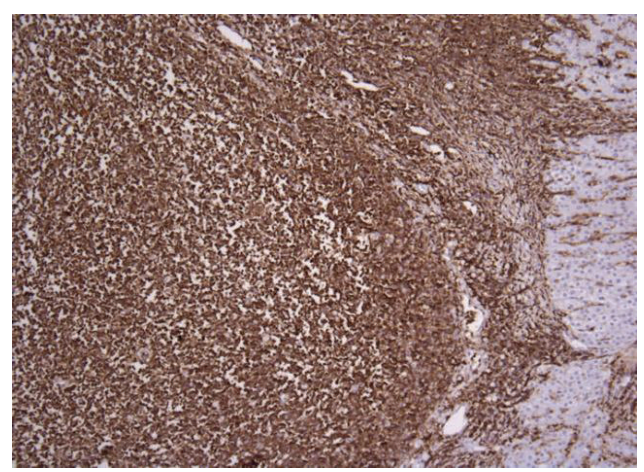

(b)

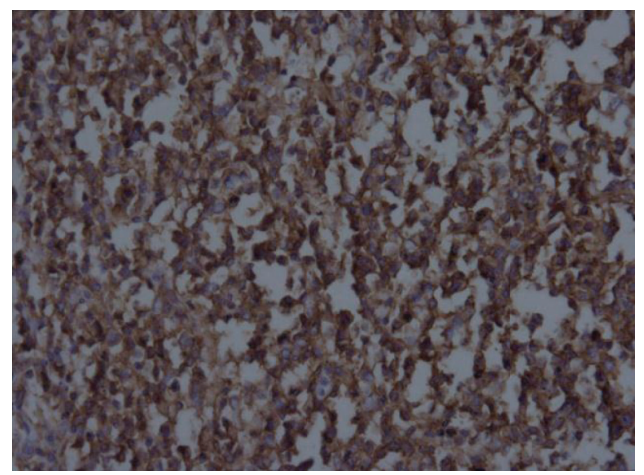

(d)

FIGURE 3: (a) Immuno.histochemistry showed that germinal centers mainly comprised CD20 (+) B lymphocytes (100x magnification) (b) Germinal centers mainly comprised LCA $(+)$ lymphocytes (100x magnification) (c) T lymphocytes in interfollicular area and surrounding germinal centers were CD45RO (+) (100x magnification) (d) Germinal centers mainly comprised CD20 (+) B lymphocytes (400x magnification).

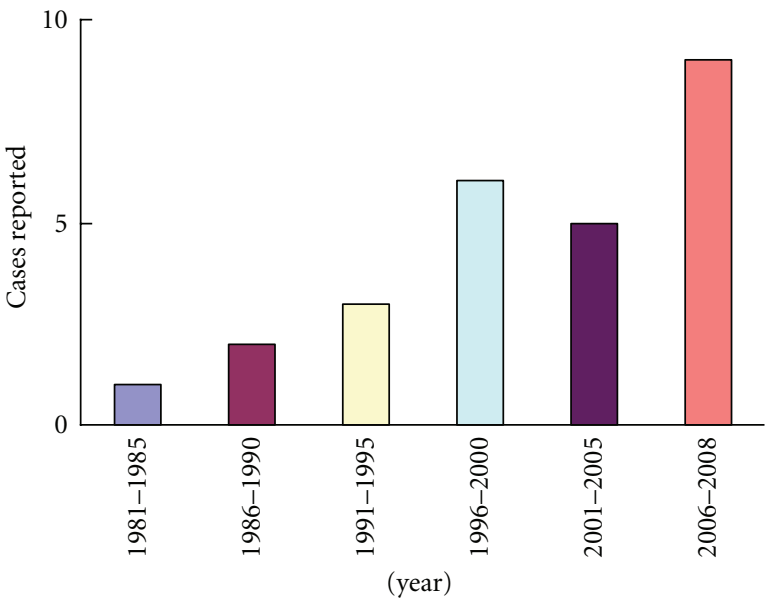

Figure 4: The incidence rate of this disorder seems to be increasing, calculated by the time when reported.

the reported have been misdiagnosed. Hepatic RLH has most frequently been misdiagnosed as HCC, as preoperative diagnosis are HCC (6/7) for our cases, while HCC (15/41) for all cases including the reported. Thus, differential diagnosis between hepatic RLH and HCC is important but difficult, given their similarity on radiological appearances. However, in our cases, we found subtle differences in radiological findings between hepatic RLH and HCC. On MRI scan, hepatic RLH is always moderately enhanced in the arterial phase (Figure 1(c)), hypodense in the portal phase (Figure 1(d)) and unclear in the delayed phase (Figure 1(e)); while HCC is remarkably enhanced in the arterial phase (Figure 1(h)), prominently hypodense in the portal phase (Figure 1(i)) and more hypodense in the delayed phase (Figure $1(\mathrm{j})$ ). The most characteristic radiological findng is that hepatic RLH becomes unclear in the delayed phase after injection of contrast agents. In addition, HCC is always accompanied by hepatitis virus infection and elevated level of AFP in China, which can be added to differential clues. From all the cases, we conclude the following radiological clues to help to diagnose hepatic RLH: (a) well-defined hypoechoic mass on the US images [4-7, 9-12, 14, 17-19, 23, 24, 32-34]; (b) low-density lesion in plain CT phase, mild enhancement in the arterial phase, hypodense areas in the portal phase $[1-7,9,10,12-18,23,24]$; (c) hypointense signal on MRI T1-weighted imaging, hyperintense signal on T2-weighted imaging, moderately enhanced in the arterial phase, hypodense areas in the portal phase and unclear in the delayed 
TABLE 1: Background data and clinical characteristics of all cases including the reported.

\begin{tabular}{|c|c|}
\hline \multicolumn{2}{|c|}{ Background data and clinical characteristics } \\
\hline Variables & Cases \\
\hline \multicolumn{2}{|l|}{ Age (Y) } \\
\hline $15-30$ & 1 \\
\hline $31-60$ & 21 \\
\hline $60-85$ & 19 \\
\hline \multicolumn{2}{|l|}{ Sex } \\
\hline Female & 36 \\
\hline Male & 5 \\
\hline \multicolumn{2}{|l|}{ Concomitant disease } \\
\hline Chronic hepatitis & 7 \\
\hline Sjögren's syndrome & 1 \\
\hline CREST syndrome & 1 \\
\hline Autoimmune thyroiditis & 5 \\
\hline Malignant tumor & 12 \\
\hline Hepatic hemangioma/FNH & 3 \\
\hline $\mathrm{PBC}$ & 4 \\
\hline $\mathrm{DM}$ & 2 \\
\hline Immunodeficiency & 2 \\
\hline \multicolumn{2}{|c|}{ Size in the greatest dimension of lesions $(\mathrm{cm})$} \\
\hline$\leqslant 4$ & 37 \\
\hline$>4$ & 4 \\
\hline \multicolumn{2}{|l|}{ Number of lesions } \\
\hline Solitary & 35 \\
\hline Multiple & 6 \\
\hline \multicolumn{2}{|l|}{ Location } \\
\hline Rt. lobe & 21 \\
\hline Lt. lobe & 13 \\
\hline Lt. lobe and Rt. lobe & 2 \\
\hline NA & 5 \\
\hline \multicolumn{2}{|l|}{ Treatment } \\
\hline Surgical resection & 34 \\
\hline Transplantation & 2 \\
\hline CNB and PEI & 1 \\
\hline CNB and observation & 3 \\
\hline Autopsy & 1 \\
\hline
\end{tabular}

Rt: right; Lt: left; Seg: segment NA: not available; PBC: primary biliary cirrhosis; FNH: focal nodular hyperplasia; DM: diabetes mellitus; PEI: percutaneous ethanol injections; CNB: core needle biopsy; NA: not available.

phase $[2,3,6,10-15,17,18,24]$; (d) hypervascularity on angiography [11]. Preoperative imaging findings of the lesions of all the cases are listed in Table 2.

It is difficult to definitely recognize hepatic RLH by routine histologic evaluation alone. Common morphologic features include a well-demarcated region, hyperplastic lymphoid follicles with active germinal centers, hyalinized trabecular structures, and lymphocytic infiltration in the portal tracts around the nodular lesion $[1,5,6,9-15,31$, 35]. However, all of these appearances are not enough to distinguish hepatic RLH from inflammatory myofibroblastic
TABLE 2: Preoperative imaging findings of hepatic RLH of all cases including the reported.

\begin{tabular}{lc}
\hline Preoperative imaging findings & Cases \\
\hline US & 7 \\
$\quad$ Hypoechoic mass & \\
CT & 18 \\
$\quad$ Plain: hypodense & 12 \\
$\quad$ Arterial: significantly/slightly/peripherally enhanced & 12 \\
$\quad$ Parenchymal and portal: clearly/vaguely low & \\
MRI & 13 \\
$\quad$ Plain T1: low; T2: high & 8 \\
$\quad \begin{array}{l}\text { Arterial: highly/slightly enhanced } \\
\quad \text { Portal and delayed: peripherally ring enhanced or }\end{array}$ & 8 \\
$\quad$ clearly/vaguely hypointense & \\
Angiography & 10 \\
$\quad$ Hypervascularity & \\
\hline $\begin{array}{l}\text { US: ultrasonographic; CT: computerized tomography; MRI: magnetic } \\
\text { resonance imaging. }\end{array}$
\end{tabular}

TABLE 3: Pathological characteristics of hepatic RLH of all cases including the reported.

\begin{tabular}{lc}
\hline $\begin{array}{l}\text { Histological, immunohistochemical, and molecular } \\
\text { findings }\end{array}$ & Cases \\
\hline LIPTANL & 22 \\
Germinal center CD20/L26 (+) & 26 \\
Germinal center LCA (+) & 6 \\
$\begin{array}{l}\text { Interfollicular area and surrounding germinal centers } \\
\text { CD45RO/UCHL1 (+) }\end{array}$ & 15 \\
Area surrounding germinal centers CD3 (+) & 11 \\
$\begin{array}{l}\text { Ductal structures at the periphery of the nodule CK7 (+) } \\
\text { Polyclonal in } \kappa \text { and } \lambda \text { light chain staining }\end{array}$ & 5 \\
$\begin{array}{l}\text { Massive infiltration of heterogeneous mature lymphoid } \\
\text { cells with no nuclear atypia, forming follicles and germinal } \\
\text { centers }\end{array}$ & 41 \\
\hline $\begin{array}{l}\text { LIPTANL: lymphocytic infiltration in the portal tracts around the nodular } \\
\text { lesion; NA: not available; L26 = CD20; UCHL1 = CD45RO. }\end{array}$
\end{tabular}

tumor and MALT lymphoma, given the similarity of morphologic features among them. Immunohistochemical and molecular genetic investigations are necessary to differentiate them [35]. In most hepatic RLH cases, germinal centers consist of polyclonal mature lymphocytes without cytologic atypia (mainly CD20 (+) and LCA (+)), and lymphocytes in the interfollicular area were predominantly CD3 $(+)$ and/or CD45RO (+) (Table 3). In addition, polyclonal patterns of $\mathrm{IgH}$ and TCR- $\gamma$ gene rearrangements of lymphocytes in the lesions were often observed (Table 3 ). However, in cases of primary hepatic MALT-type NHL [36-43], small B cells proliferating in lymphoid follicles displayed monoclonality. Although preoperative needle biopsy was applied in 5 reported cases $[15,17,20,35]$, it is not recommended because it cannot provide comprehensive details of results of immunohistochemical and molecular genetic studies. Also it 
is difficult to differentiate hepatic RLH from primary hepatic lymphoma only by needle biopsy [35].

Although hepatic RLH is generally thought to be benign, it might transform into malignant lymphoma [32], as reported in the lung [44] and stomach [45]. These early reports, however, were lack of the use of immunofluorescent and molecular techniques, and it is likely that these cases were in fact the early stage of primary lymphoma misdiagnosed as benign [46]. But evidence of progression from histologically benign, immunohistochemically polyclonal lymphoid infiltrates to malignant lymphoma in cutaneous pseudolymphoma has been reported in the literature [47]. Also we speculate hepatic RLH may grow with time, because the lesion of the male patient progressed during the three years before operation, similar to two reported cases $[11,18]$. But different voices existed that hepatic RLH might have spontaneous regression, as in three cases [20,35], diameter of the lesion had decreased during the followup without operation. However, it is not convincible because hepatic RLH was diagnosed by core needle biopsy in the three cases. Surgical resection is suggested both for treatment and for a definitive diagnosis of hepatic RLH even when pseudolymphoma is diagnosed preoperatively. Treatments for all the cases were surgical resection (7/7) in our series; while surgical resection (33/41), liver transplantation (2/41), percutaneous ethanol injection (1/41), core needle biopsy and observation (3/41) $[20,35]$, and unknown $(2 / 41)$ in all cases including the reported. No complication related to surgical treatment happened. The prognosis of hepatic RLH is good, and all the patients treated by resection have shown no recurrence or progression to lymphoma.

In conclusion, hepatic RLH is a rare disease which mostly occurs in females, and the exact etiology of this disease is still unknown. Hepatic RLH has similar features with malignant liver tumors on radiological findings, but subtle differences such as "the lesion becomes unclear in the delayed phase on MRI scan with injection of contrast agents" can be found to be helpful for differential diagnosis. An accurate postoperative diagnosis of this disease depends not only on morphologic findings, but also on immunohistochemical analysis and molecular investigations. Given that hepatic RLH may grow with time or might even transform into malignant lymphoma, surgical resection is suggested, both for the safety and for good prognosis of the disease.

\section{Conflict of Interests}

L. Yuan, Y. Zhang, Y. Wang, W. Cong, and M. Wu have no conflict of interests or financial ties to disclose.

\section{References}

[1] S. Sharifi, M. Murphy, M. Loda, G. S. Pinkus, and U. Khettry, "Nodular lymphoid lesion of the liver: an immune-mediated disorder mimicking low-grade malignant lymphoma," American Journal of Surgical Pathology, vol. 23, no. 3, pp. 302-308, 1999.

[2] K. Willenbrock, S. Kriener, S. Oeschger, and M. L. Hansmann, "Nodular lymphoid lesion of the liver with simultaneous focal nodular hyperplasia and hemangioma: discrimination from primary hepatic MALT-type non-Hodgkin's lymphoma," Virchows Archiv, vol. 448, no. 2, pp. 223-227, 2006.

[3] V. Grouls, "Pseudolymphoma (inflammatory pseudotumor) of the liver," Zentralblatt fur Allgemeine Pathologie und Pathologische Anatomie, vol. 133, no. 6, pp. 565-568, 1987.

[4] T. Ohtsu, Y. Sasaki, H. Tanizaki et al., "Development of pseudolymphoma of liver following interferon-alpha therapy for chronic hepatitis B," Internal Medicine, vol. 33, no. 1, pp. 1822, 1994.

[5] K. Katayanagi, T. Terada, Y. Nakanuma, and T. Ueno, "A case of pseudolymphoma of the liver," Pathology International, vol. 44, no. 9, pp. 704-711, 1994.

[6] S. R. Kim, Y. Hayashi, K. B. Kang et al., "A case of pseudolymphoma of the liver with chronic hepatitis C," Journal of Hepatology, vol. 26, no. 1, pp. 209-214, 1997.

[7] H. Okubo, H. Maekawa, K. Ogawa et al., "Pseudolymphoma of the liver associated with Sjögren's syndrome," Scandinavian Journal of Rheumatology, vol. 30, no. 2, pp. 117-119, 2001.

[8] D. C. Snover, A. H. Filipovich, L. P. Dehner, and W. Krivit, "'Pseudolymphoma'. A case associated with primary immunodeficiency disease and polyglandular failure syndrome," Archives of Pathology and Laboratory Medicine, vol. 105, no. 1, pp. 46-49, 1981.

[9] H. Isobe, S. Sakamoto, H. Sakai et al., "Reactive lymphoid hyperplasia of the liver," Journal of Clinical Gastroenterology, vol. 16, no. 3, pp. 240-244, 1993.

[10] H. Takahashi, H. Sawai, Y. Matsuo et al., "Reactive lymphoid hyperplasia of the liver in a patient with colon cancer: report of two cases," BMC Gastroenterology, vol. 6, article 25, 2006.

[11] K. Nagano, Y. Fukuda, I. Nakano et al., "Case report: reactive lymphoid hyperplasia of liver coexisting with chronic thyroiditis: radiographical characteristics of the disorder," Journal of Gastroenterology and Hepatology, vol. 14, no. 2, pp. 163-167, 1999.

[12] N. Maehara, K. Chijiiwa, I. Makino et al., "Segmentectomy for reactive lymphoid hyperplasia of the liver: report of a case," Surgery Today, vol. 36, no. 11, pp. 1019-1023, 2006.

[13] K. Sato, Y. Ueda, M. Yokoi, K. Hayashi, T. Kosaka, and S. Katsuda, "Reactive lymphoid hyperplasia of the liver in a patient with multiple carcinomas: a case report and brief review," Journal of Clinical Pathology, vol. 59, no. 9, pp. 990992, 2006.

[14] T. Tanizawa, Y. Eishi, R. Kamiyama et al., "Reactive lymphoid hyperplasia of the liver characterized by an angiofollicular pattern mimicking Castleman's disease," Pathology International, vol. 46, no. 10, pp. 782-786, 1996.

[15] K. Shiozawa, H. Kinoshita, H. Tsuruta et al., "A case of pseudolymphoma of the liver diagnosed before operation," Nipponn Syoukakibyou Gakkai Zassi, vol. 101, no. 7, pp. 772-778, 2004.

[16] H. S. Park, K. Y. Jang, Y. K. Kim, H. C. Baik, and S. M. Woo, "Histiocyte-rich reactive lymphoid hyperplasia of the liver: unusual morphologic features," Journal of Korean Medical Science, vol. 23, no. 1, pp. 156-160, 2008.

[17] N. Matsumoto, M. Ogawa, M. Kawabata et al., "Pseudolymphoma of the liver: sonographic findings and review of the literature," Journal of Clinical Ultrasound, vol. 35, no. 5, pp. 284-288, 2007.

[18] T. Okada, H. Mibayashi, K. Hasatani et al., "Pseudolymphoma of the liver associated with primary biliary cirrhosis: a case report and review of literature," World Journal of Gastroenterology, vol. 15, no. 36, pp. 4587-4592, 2009. 
[19] T. Machida, T. Takahashi, T. Itoh, M. Hirayama, T. Morita, and S. Horita, "Reactive lymphoid hyperplasia of the liver: a case report and review of literature," World Journal of Gastroenterology, vol. 13, no. 40, pp. 5403-5407, 2007.

[20] H. Ota, N. Isoda, F. Sunada et al., "A case of hepatic pseudolymphoma observed without surgical intervention," Hepatology Research, vol. 35, no. 4, pp. 296-301, 2006.

[21] R. Jiménez, A. Beguiristain, I. Ruiz-Montesinos, F. Garnateo, and M. Echenique Elizondo, "Nodular lymphoid hyperplasia of the liver. Pseudolymphoma," Revista Española de Enfermedades Digestivas, vol. 99, no. 10, pp. 299-306, 2007.

[22] E. Lin, "Reactive lymphoid hyperplasia of the liver identified by FDG PET," Clinical Nuclear Medicine, vol. 33, no. 6, pp. 419-420, 2008.

[23] K. Yoshikawa, M. Konisi, T. Kinoshita et al., "Reactive lymphoid hyperplasia of the liver: literature review and 3 case reports," Hepato-Gastroenterology, vol. 58, no. 109, pp. 13491353, 2011.

[24] J. Tuckett, M. Hudson, S. White, and J. Scott, "Reactive lymphoid hyperplasia of the liver: a case report and review of imaging characteristics," European Journal of Radiology Extra, vol. 79, no. 1, pp. e11-e14, 2011.

[25] E. Sabattini, K. Bisgaard, S. Ascani et al., "The EnVision++ system: a new immunohistochemical method for diagnostics and research. Critical comparison with the APAAP, ChemMate(TM), CSA, LABC, and SABC techniques," Journal of Clinical Pathology, vol. 51, no. 7, pp. 506-511, 1998.

[26] S. L. Abbondanzo and L. H. Sobin, "Gastric "pseudolymphoma": a retrospective morphologic and immunophenotypic study of 97 cases," Cancer, vol. 79, no. 9, pp. 1656-1663, 1997.

[27] D. M. Knowles, F. A. Jakobiec, L. McNally, and J. S. Burke, "Lymphoid hyperplasia and malignant lymphoma occurring in the ocular adnexa (orbit, conjunctiva, and eyelids): a prospective multiparametric analysis of 108 cases during 1977 to 1987," Human Pathology, vol. 21, no. 9, pp. 959-973, 1990.

[28] S. L. Abbondanzo, W. Rush, K. E. Bijwaard, and M. N. Koss, "Nodular lymphoid hyperplasia of the lung: a clinicopathologic study of 14 cases," American Journal of Surgical Pathology, vol. 24, no. 4, pp. 587-597, 2000.

[29] M. F. Baldassano, E. M. Bailey, J. A. Ferry, N. L. Harris, and L. M. Duncan, "Cutaneous lymphoid hyperplasia and cutaneous marginal zone lymphoma: comparison of morphologic and immunophenotypic features," American Journal of Surgical Pathology, vol. 23, no. 1, pp. 88-96, 1999.

[30] Y. Mizukami, N. Ikuta, T. Hashimoto et al., "Pseudolymphoma of the thyroid," Acta Pathologica Japonica, vol. 38, no. 10, pp. 1329-1336, 1988.

[31] L. Pantanowitz, P. F. Saldinger, and M. E. Kadin, "Pathologic quiz case: hepatic mass in a patient with renal cell carcinoma," Archives of Pathology and Laboratory Medicine, vol. 125, no. 4, pp. 577-578, 2001.

[32] S. I. Sato, T. Masuda, H. Oikawa et al., "Primary hepatic lymphoma associated with primary biliary cirrhosis," American Journal of Gastroenterology, vol. 94, no. 6, pp. 1669-1673, 1999.

[33] A. Nonomura, H. Minato, K. Shimizu et al., "Pseudolymphoma (reactive lymphoid hyperplasia) of the liver containing epithelioid cell granulomas and Schaumann's bodies in giant cells: a case report," International Journal of Surgical Pathology, vol. 6, no. 2, pp. 101-108, 1998.

[34] R. Jiménez, A. Beguiristain, I. Ruiz-Montesinos et al., "Image of the month: reactive lymphoid hyperplasia," Archives of Surgery, vol. 143, no. 8, pp. 805-806, 2008.
[35] Y. Zen, T. Fujii, and Y. Nakanuma, "Hepatic pseudolymphoma: a clinicopathological study of five cases and review of the literature," Modern Pathology, vol. 23, no. 2, pp. 244-250, 2010.

[36] C. J. Story, A. A. Morley, D. R. Turner, and R. Seshadri, "Diagnostic use of immunoglobulin and T-cell receptor gene rearrangements in lymphoproliferative disease," Australian and New Zealand Journal of Medicine, vol. 17, no. 1, pp. 1-8, 1987.

[37] P. G. Isaacson, P. M. Banks, P. V. Best, S. P. McLure, H. K. Muller-Hermelink, and J. I. Wyatt, "Primary low-grade hepatic B-cell lymphoma of mucosa-associated lymphoid tissue (MALT)-type," American Journal of Surgical Pathology, vol. 19, no. 5, pp. 571-575, 1995.

[38] J. P. Bronowicki, C. Bineau, P. Feugier et al., "Primary lymphoma of the liver: clinical-pathological features and relationship with HCV infection in French patients," Hepatology, vol. 37, no. 4, pp. 781-787, 2003.

[39] C. M. Kirk, D. Lewin, and J. Lazarchick, "Primary hepatic B-cell lymphoma of mucosa-associated lymphoid tissue," Archives of Pathology and Laboratory Medicine, vol. 123, no. 8, pp. 716-719, 1999.

[40] M. Maes, C. Depardieu, J. L. Dargent et al., "Primary lowgrade B-cell lymphoma of malt-type occurring in the liver: a study of two cases," Journal of Hepatology, vol. 27, no. 5, pp. 922-927, 1997.

[41] R. M. Prabhu, L. J. Medeiros, D. Kumar et al., "Primary hepatic low-grade B-cell lymphoma mucosa-associated lymphoid tissue (MALT) associated with primary biliary cirrhosis," Modern Pathology, vol. 11, no. 4, pp. 404-410, 1998.

[42] M. Q. Ye, A. Suriawinata, C. Black, A. D. Min, J. Strauchen, and S. N. Thung, "Primary hepatic marginal zone B-cell lymphoma of mucosa-associated lymphoid tissue type in a patient with primary biliary cirrhosis," Archives of Pathology and Laboratory Medicine, vol. 124, no. 4, pp. 604-608, 2000.

[43] E. Zucca, A. Conconi, E. Pedrinis et al., "Nongastric marginal zone B-cell lymphoma of mucosa-associated lymphoid tissue," Blood, vol. 101, no. 7, pp. 2489-2495, 2003.

[44] M. N. Koss, L. Hochholzer, and P. W. Nichols, "Primary nonHodgkin's lymphoma and pseudolymphoma of lung: a study of 161 patients," Human Pathology, vol. 14, no. 12, pp. 10241038, 1983.

[45] J. J. Brooks and H. T. Enterline, "Gastric pseudolymphoma. Its three subtypes and relation to lymphoma," Cancer, vol. 51, no. 3, pp. 476-486, 1983.

[46] E. A. Holland, G. G. Ghahremani, W. A. Fry, and T. A. Victor, "Evolution of pulmonary pseudolymphomas: clinical and radiologic manifestations," Journal of Thoracic Imaging, vol. 6 , no. 4, pp. 74-80, 1991.

[47] B. F. Kulow, H. Cualing, P. Steele et al., "Progression of cutaneous B-cell pseudolymphoma to cutaneous B-cell lymphoma," Journal of Cutaneous Medicine and Surgery, vol. 6, no. 6, pp. 519-528, 2002. 


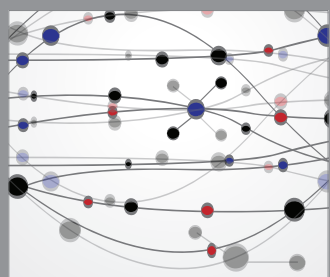

The Scientific World Journal
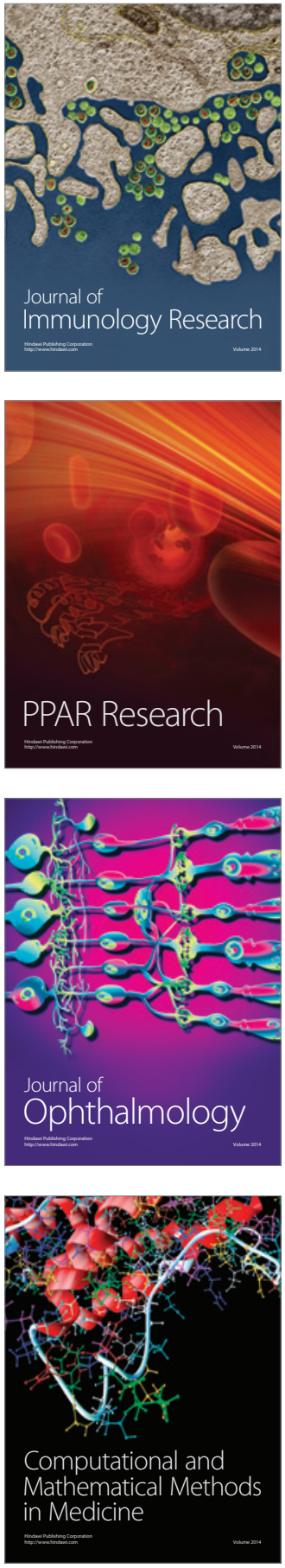

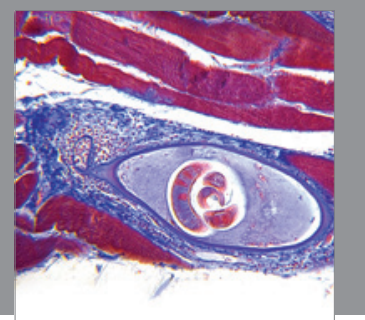

Gastroenterology

Research and Practice
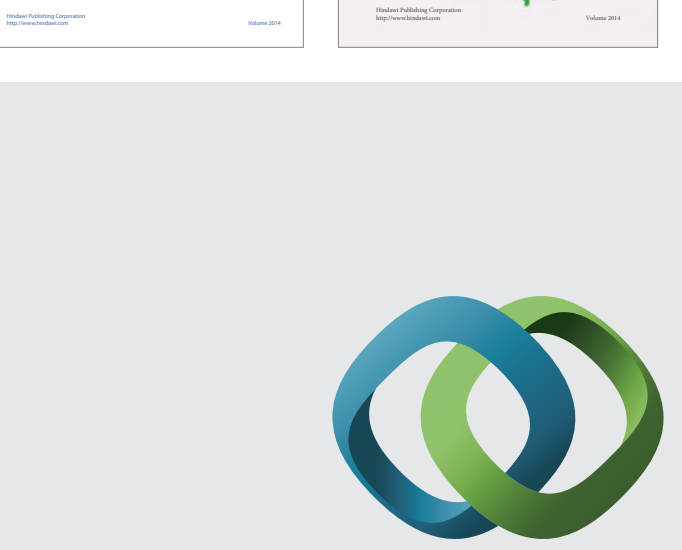

\section{Hindawi}

Submit your manuscripts at

http://www.hindawi.com
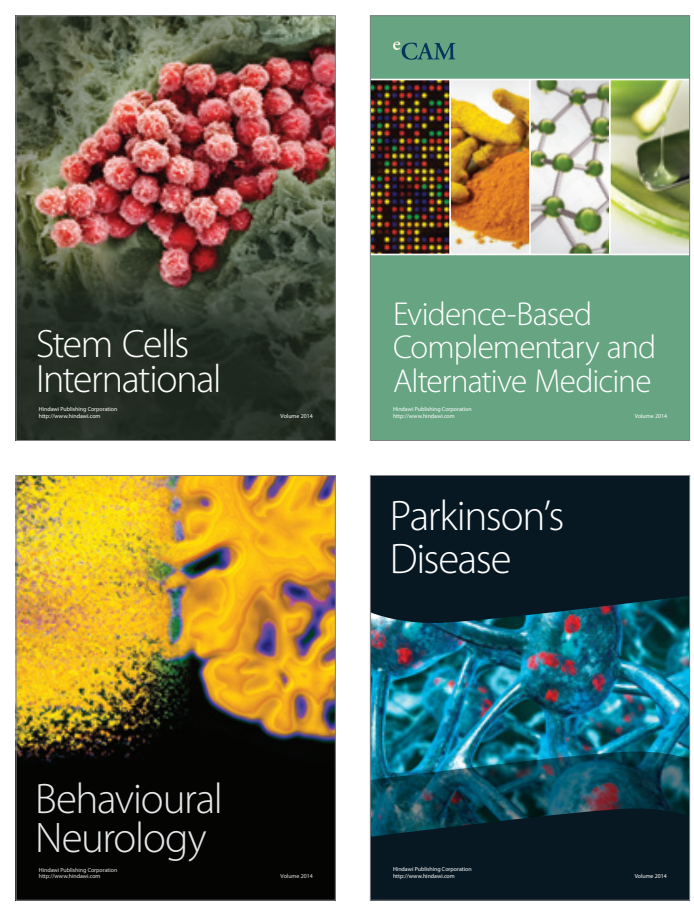

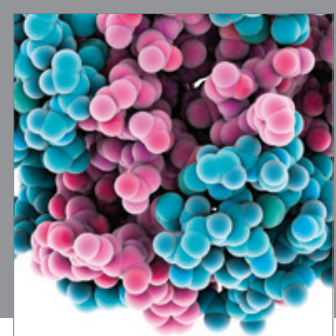

Journal of
Diabetes Research

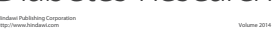

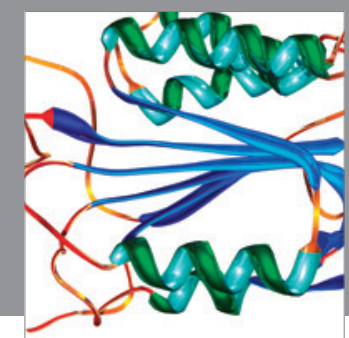

Disease Markers
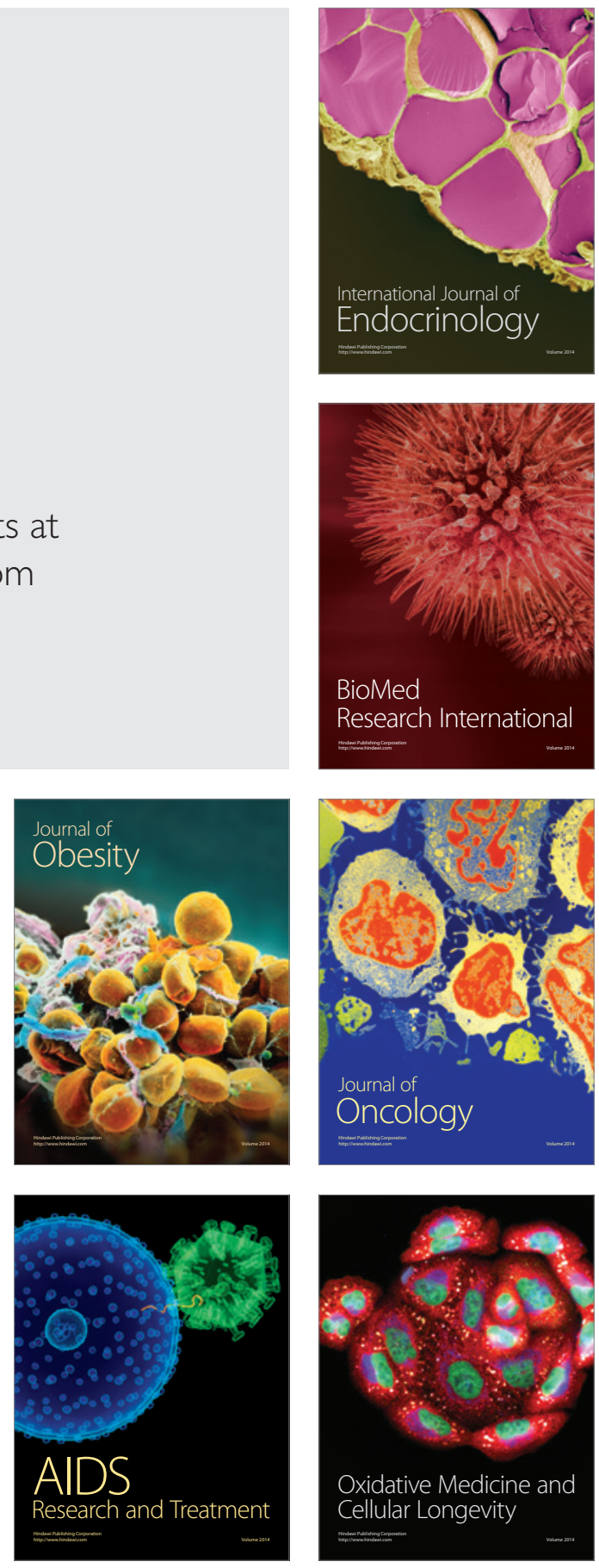